\title{
Urinary orosomucoid and retinol binding protein levels as early diagnostic markers for diabetic nephropathy
}

\section{Xue-hong Zhou ( $\nabla$ m18639186869@163.com )}

The First Affiliated Hospital of Henan Polytechnic University

Shu-yan Liu

First Affiliated Hospital of Henan Polytechnic University

\section{Lin Feng}

Laboratory of Jiaozuo Customs

\section{Bo Yang}

First Affiliated Hospital of Henan Polytechnic University

\section{Yong-feng Li}

First Affiliated Hospital of Henan Polytechnic University

\section{Xiao-qing Wang}

First Affiliated Hospital of Henan Polytechnic University

\section{Jie Chen}

First Affiliated Hospital of Henan Polytechnic University

Hui-hui Wang

First Affiliated Hospital of Henan Polytechnic University

\section{Research article}

Keywords: inflammatory responses, protein markers, diagnosis, urine microalbumin; clinical research

Posted Date: October 26th, 2020

DOl: https://doi.org/10.21203/rs.3.rs-38721/v2

License: (c) (i) This work is licensed under a Creative Commons Attribution 4.0 International License. Read Full License 


\section{Abstract}

\section{Background:}

Diagnosing diabetic nephropathy is important to prevent long-term kidney damage and determine the prognosis of patients with diabetes. Since some kidney injury biomarkers increase in the early stages of diabetic nephropathy, this study investigated the clinical significance of combined detection of urine orosomucoid and retinol-binding protein for early diagnosis of diabetic nephropathy.

\section{Methods:}

We included 72 patients with type 2 diabetes and 34 healthy persons between August 2016 and July 2018 at our hospital. Using the Mogensen grading criteria, participants were classified as having diabetes or diabetic nephropathy, and healthy persons constituted the control group. Urine orosomucoid and retinol-binding protein levels were measured and correlated with other variables.

\section{Results:}

Increase in renal damage raised urinary orosomucoid levels gradually $(P<0.05)$. Urinary retinol-binding protein and microalbumin levels were significantly higher in the diabetes group than in control and nephropathy groups. Orosomucoid and retinol-binding protein might be independent risk factors for diabetes and diabetic nephropathy. Urinary orosomucoid significantly correlated with retinol-binding protein and microalbumin levels ( $r=0.489$ and 0.513 , respectively) in the diabetic nephropathy group. The receiver operating characteristic curve yielded a sensitivity, specificity, and correction index of 0.941 , 0.842 , and 0.783 , respectively, while analysis for retinol-binding protein yielded a sensitivity, specificity, and correction index of $0.942,1.000$, and 0.941 , respectively.

\section{Conclusion:}

The increase in urine orosomucoid and retinol-binding protein levels can be detected in the early stages of type 2 diabetic nephropathy. Therefore, both markers are important for diabetic nephropathy detection and early treatment.

\section{Background}

Diabetic nephropathy (DN) is the most common and severe chronic vascular complication among patients with type 2 diabetes mellitus (T2DM). (1) It leads to chronic renal failure and is the leading cause of death due to diabetes. However, DN often occurs with no obvious symptoms in the early stage. Common diagnostic indicators of DN include 24-hour urine microalbumin (MAL), urea nitrogen, and serum creatinine levels. However, they can be affected by many factors, such as urinary tract or systemic infections, strenuous exercise, bleeding, or drugs that affect the kidneys. (2) The accuracy and specificity of these indicators are not high, and they have limitations (3); thus, more research is needed to identify newer, more accurate, and specific early diagnostic markers of DN. Presently, preliminary progress has 
been made in kidney disease research using proteomics technology. Diabetic urine proteome research has shown that some protein markers have a predictive value in early DN. $(4,5)$ Jang $(6)$ used twodimensional gel electrophoresis (2-DE) and two-dimensional fluorescence difference gel electrophoresis (2D-DIGE) to analyze the urine of patients with T2DM presenting microalbuminuria, macroalbuminuria, or normal proteinuria and that of healthy volunteers; the specimens were further analyzed using comparative proteomics. Screening for differentially expressed proteins in the urine of DN patients, performing mass spectrometry and bioinformatics analysis of the differential proteins, and selecting and obtaining six differential proteins such as orosomucoid and retinol-binding protein (RBP) for research will help in the early diagnosis of DN. El-Beblawy et al. (7) assessed serum and urinary orosomucoid levels in children and adolescents with type 1 diabetes and suggested that orosomucoid is a significant independent factor for diabetic microvascular complications and can be considered as an early marker of renal injury. Wang et al. (8) evaluated the value of urinary orosomucoid in early renal impairment screening in T2DM patients and showed that, the orosomucoid level had high diagnostic efficiency to aid in the early detection of renal impairment in T2DM patients. Mahfouz, Assiri and Mukhtar (9) suggested that the RBP4 marker may serve as a tool to follow-up on the development and progression of DN. In this study, the urine orosomucoid and RBP levels were measured in healthy people, patients with T2DM, and patients with early DN. The differences between the three groups were compared. They also assessed the clinical significance of these urine orosomucoid and RBP levels in the diagnosis of early type 2 DN and their clinical value to monitor the progression of nephropathy.

\section{Methods}

\section{Study subjects}

Thirty-four healthy people who underwent physical examination at our hospital between August 2016 and July 2018 were categorized as the normal control (NC) group, including 18 males and 16 females, with an average age of $47.9 \pm 14.2$ years. Seventy-seven patients with T2DM who were hospitalized at the same time were assessed according to the Mogensen classification criteria for the degree of kidney damage. These patients were categorized as those with T2DM (T2DM group; $n=38$; microalbumin (MAL) $<30$ $\mathrm{mg} / 24 \mathrm{~h}$ ), which included 21 males and 17 females, with an average age of $48.7 \pm 13.6$ years, and patients with early DN and combined diabetic retinopathy (T2DN group; $n=34 ; M A L 30-300 \mathrm{mg} / 24 \mathrm{~h}$ ), which included 19 males and 15 females, with an average age of $49.1 \pm 14.4$ years. Diabetes was diagnosed and classified according to the 1999 diagnostic criteria of the World Health Organization. (10) The exclusion criteria were diabetic ketosis, hyperglycemia and osmotic pressure syndrome, combined fever and infection, acute cardiocerebrovascular diseases and urinary system diseases (kidney stones, acute and chronic nephritis, and nephrotic syndrome), non-diabetic congestive heart failure, liver dysfunction, rheumatic diseases, hematological diseases, pregnancy, tumors, fractures, primary hyperparathyroidism, a history of kidney transplant and intake of glucocorticoids, history of immunosuppressant and nephrotoxic drugs use, history of renal damage caused by strenuous exercise, and severe hypertension.

\section{Data collection}


We recorded the medical history of all patients and measured their diastolic blood pressure (DBP), systolic blood pressure (SBP), height $(\mathrm{cm})$, and weight $(\mathrm{kg})$. The levels of total cholesterol (CHOL), lowdensity lipoprotein (LDL) CHOL, triglycerides (TG), fasting blood glucose (FBG), 2-hour blood glucose, serum creatinine ( $\mathrm{SCr}$ ), urine orosomucoid, and RBP were measured using Applied blood biochemical detector (Hitachi 7600, Hitachi, Japan). HLC-723 HbA1c Analyzer (Japan Toshiba, Tokyo, Japan), i.e., high-pressure liquid phase ion-exchange chromatography was used to determine glycosylated hemoglobin ( $\mathrm{HbA} 1 \mathrm{c}$ ) levels. The urine specimen was collected between $10 \mathrm{pm}$ and $6 \mathrm{am}$, and the total urine volume $(\mathrm{mL})$ was recorded after mixing. IMMAGE 800 protein chemistry analyzer (Beckman Coulter, USA) was used to detect urine MAL. The urine orosomucoid level was determined using the rate scattering immunoturbidimetric method performed on the Array 360 System (Beckman Coulter, USA). Orosomucoid reagents, calibration cards, and calibration were provided by the manufacturer. The detection temperature was $37^{\circ} \mathrm{C}$, while the measurement wavelength of the instrument was $340 \mathrm{~nm}$; the immunoturbidimetric measurement was performed after calibration. The Chronic Kidney Disease Epidemiology Collaboration (CKD-EPI) equation (11) was used to evaluate the estimated glomerular filtration rate (eGFR).

\section{Statistical analysis}

For normally distributed data determined using the Shapiro-Wilk's test, the indicators in each group were expressed as the mean \pm standard deviation. The Chi-square test was used to compare quantitative data between groups. The mean values for each of the three groups were compared using one-way analysis of variance. If there were significant differences between the groups, intra-group comparisons were performed using the least significant difference. A binary logistic regression model was used to determine the factors associated with T2DM and T2DN, and correlation analyses were performed using the Spearman's rank correlation. The receiver operating characteristic (ROC) curve was used to analyze the diagnostic points and diagnostic value of orosomucoid and RBP in DN. All statistical analyses were performed using SPSS version 23.0 (IBM Corp., Armonk, NY, USA). A two-tailed test with $P<0.05$ was considered significant.

\section{Results}

There were no significant differences in age and gender among the three groups $(P>0.05)$. There were no significant differences in DBP, BMI, CHOL, TG, and LDL levels among the three groups $(P>0.05)($ Table 1$)$.

Table 1. Comparison of general clinical data among the three groups 


\begin{tabular}{|llllll|}
\hline Groups & NC $(n=34)$ & T2DM $(n=38)$ & T2DN $(n=34)$ & $F$ & P-value \\
\hline BMI, $\mathrm{kg} / \mathrm{m} 2$ & $26.19 \pm 1.28$ & $26.32 \pm 1.31$ & $26.27 \pm 1.24$ & 0.094 & 0.911 \\
\hline SBP, $\mathrm{mmHg}$ & $117.82 \pm 12.06$ & $130.61 \pm 12.44 \mathrm{a}$ & $142.55 \pm 14.78 \mathrm{ab}$ & 33.11 & $<0.001$ \\
\hline $\mathrm{DBP}, \mathrm{mmHg}$ & $80.48 \pm 9.16$ & $83.26 \pm 9.36$ & $85.19 \pm 10.08$ & 2.098 & 0.128 \\
\hline $\mathrm{HbA} 1 \mathrm{c}(\%)$ & $5.3 \pm 0.51$ & $10.45 \pm 2.65 \mathrm{a}$ & $10.27 \pm 2.77 \mathrm{a}$ & 58.55 & $<0.001$ \\
\hline $\mathrm{FBG}, \mathrm{mmol} / \mathrm{L}$ & $4.63 \pm 0.52$ & $9.98 \pm 4.63 \mathrm{a}$ & $10.69 \pm 4.27 \mathrm{a}$ & 27.710 & $<0.001$ \\
\hline $\mathrm{CHOL}, \mathrm{mmol} / \mathrm{L}$ & $4.35 \pm 0.66$ & $4.82 \pm 1.08$ & $4.83 \pm 1.14$ & 2.670 & 0.074 \\
\hline $\mathrm{TG}, \mathrm{mmol} / \mathrm{L}$ & $1.29 \pm 0.54$ & $1.3 \pm 0.61$ & $1.33 \pm 0.56$ & 0.045 & 0.956 \\
\hline $\mathrm{LDL}, \mathrm{mmol} / \mathrm{L}$ & $2.17 \pm 0.43$ & $2.33 \pm 0.61$ & $2.41 \pm 0.69$ & 1.474 & 0.234 \\
\hline
\end{tabular}

${ }^{a}$ Compared with NC: $\mathrm{P}<0.05$,

${ }^{\mathrm{b}}$ Compared with T2DM: $\mathrm{P}<0.05$

Abbreviations: BMI, body mass index; SBP, systolic blood pressure; DBP, diastolic blood pressure; HbA1c (\%), glycosylated hemoglobin; FBG, mmol/L, fasting blood glucose; $\mathrm{CHOL}, \mathrm{mmol} / \mathrm{L}$, cholesterol; TG, $\mathrm{mmol} / \mathrm{L}$, triglycerides; LDL, mmol/L, low-density lipoproteins.

There were significant differences in SBP, $\mathrm{HbA1C}$, and FBG between the NC and the other two groups $(P<0.05)$. However, there was no significant difference in the general clinical data between the T2DM and T2DN groups $(P>0.05)$. As renal damage increased in patients, urine orosomucoid levels gradually increased as well $(P<0.05)$ (Table 2$)$.

Table 2. Comparison of urinary orosomucoid, RBP, MAL, and eGFR levels among the three groups

\begin{tabular}{|llllll|}
\hline Groups & NC $(n=34)$ & T2DM $(n=38)$ & T2DN $(n=34)$ & $F$ & P-value \\
\hline Orosomucoid, mg/L & $9.45 \pm 2.03$ & $18.35 \pm 4.04 a$ & $29.46 \pm 6.13 \mathrm{ab}$ & 177.82 & $<0.001$ \\
\hline RBP, $\mathrm{mg} / \mathrm{L}$ & $0.26 \pm 0.07$ & $0.31 \pm 0.09$ & $0.95 \pm 0.28 \mathrm{ab}$ & 172.56 & $<0.001$ \\
\hline eGFR, $\mathrm{mL} / \mathrm{min} / 1.73 \mathrm{~m} 2$ & $108.08 \pm 13.73$ & $102.17 \pm 10.12$ & $94.92 \pm 10.57^{\mathrm{ab}}$ & 11.11 & $<0.001$ \\
\hline MAL, mg/24 h & $10.22 \pm 6.42$ & $12.13 \pm 7.83$ & $199.65 \pm 49.72 \mathrm{ab}$ & 492.19 & $<0.001$ \\
\hline
\end{tabular}

${ }^{a}$ Compared with NC group: $\mathrm{P}<0.05$

${ }^{\text {b}}$ Compared with T2DM group: $\mathrm{P}<0.05$

Abbreviations, RBF, renal blood flow; eGFR, estimated glomerular filtration rate; MAL, microalbumin. 
Urine RBP and MAL levels in the T2DN group were significantly higher than those in the NC and T2DN groups $(P<0.001)$. The eGFR levels in the T2DN group were significantly lower than those in the $N C$ and T2DM groups $(P<0.001)$. There were no significant differences in RBP, eGFR, and MAL levels between the NC and T2DM groups $(P>0.05)$.

For the NC group and T2DM group, the dependent variable was whether T2DM had occurred (Yes = 1, No $=0$ ) and the independent variables were the four variables (SBP, HbA1c, FBG, and orosomucoid) with differences between the two groups, as shown in Tables 1 and 2. A binary logistic regression model was established and used to determine the influence of these four variables on T2DM (Table 3), and all were shown to be risk factors (all OR> $1, p<0.05$ ).

Table 3. Binary logistic regression analysis of the factors associated with type 2 diabetes mellitus

\begin{tabular}{|llllll|}
\hline Variable & $\begin{array}{l}\text { Regression } \\
\text { coefficient (B) }\end{array}$ & $\begin{array}{l}\text { Significance } \\
\text { level }(\mathrm{P})\end{array}$ & $\begin{array}{l}\text { Odds ratio } \\
(\mathrm{OR})\end{array}$ & $\begin{array}{l}95 \% \mathrm{Cl} \text { of the } \\
\text { OR }\end{array}$ \\
\hline Lower limit & $\begin{array}{l}\text { Upper } \\
\text { limit }\end{array}$ & & & & \\
\hline SBP, mmHg & 0.106 & 0.000 & 1.112 & 1.056 & 1.170 \\
\hline HbA1c (\%) & 2.133 & 0.001 & 8.438 & 2.320 & 30.688 \\
\hline FBG, mmol/L & 1.022 & 0.000 & 2.779 & 1.663 & 4.646 \\
\hline $\begin{array}{l}\text { Orosomucoid, } \\
\text { mg/L }\end{array}$ & 0.964 & 0.001 & 2.621 & 1.521 & 4.516 \\
\hline
\end{tabular}

Abbreviations: SBP, systolic blood pressure; HbA1c, glycosylated hemoglobin; FBG, fasting blood glucose.; $\mathrm{OR}$, odds ration; $\mathrm{Cl}$, confidence interval.

For the T2DM group and T2DN group, the dependent variable was whether T2DN had occurred ( $Y e s=1$, $\mathrm{No}=0$ ) and the independent variables were the five variables with differences between the two groups, as shown in Tables 1 and 2. A binary logistic regression model was established for analysis (Table 4).

Table 4. Binary logistic regression analysis of the factors associated with Type 2 diabetic nephropathy 


\begin{tabular}{|c|c|c|c|c|c|}
\hline Variable & $\begin{array}{l}\text { Regression } \\
\text { coefficient (B) }\end{array}$ & $\begin{array}{l}\text { Significance } \\
\text { level }(P)\end{array}$ & $\begin{array}{l}\text { Odds ratio } \\
\text { (OR) }\end{array}$ & $\begin{array}{l}95 \% \mathrm{Cl} \text { of } \\
\text { the OR }\end{array}$ & \\
\hline Lower limit & Upper limit & & & & \\
\hline SBP, mmHg & 0.089 & 0.000 & 1.093 & 1.045 & 1.143 \\
\hline $\begin{array}{l}\text { Orosomucoid, } \\
\mathrm{mg} / \mathrm{L}\end{array}$ & 0.626 & 0.000 & 1.871 & 1.360 & 2.574 \\
\hline RBP, mg/L & 0.241 & 0.023 & 13.305 & 9.079 & 26.000 \\
\hline $\begin{array}{l}\text { eGFR, } \\
\mathrm{mL} / \mathrm{min} / 1.73 \mathrm{~m}^{2}\end{array}$ & -0.054 & 0.021 & 0.948 & 0.905 & 0.992 \\
\hline $\mathrm{MAL}, \mathrm{mg} / 24 \mathrm{~h}$ & 0.892 & 0.000 & 2.441 & 1.070 & 3.149 \\
\hline
\end{tabular}

Abbreviations: RBF, renal blood flow; eGFR, estimated glomerular filtration rate; MAL, microalbumin; SBP, systolic blood pressure; OR, odds ration; $\mathrm{Cl}$, confidence interval.

Of the five factors that were included in the regression model $(p<0.05)$, SBP, orosomucoid, RBP, and MAL were all determined to be risk factors $(O R>1)$, and eGFR was shown to be a protective factor $(O R=0.948>$ 1). Correlation analysis showed that in the T2DN group, the urinary orosomucoid level was significantly positively correlated with RBP $(r=0.489)$ and MAL $(r=0.513)$. RBP and MAL were significantly positively correlated with a correlation coefficient of 0.468 . eGFR and urine orosomucoid, RBP, and MAL were significantly negatively correlated ( $r=-0.577,-0.474$, and -0.466 , respectively).

ROC curve analysis was used to assess the diagnostic points and diagnostic value of orosomucoid and that of RBP to predict DN. Figure 1 and Table 5 show the areas under the ROC curves for orosomucoid and RBP with the respective standard error values.

Table 5. Areas under the two ROC curves for predicting diabetic nephropathy

\begin{tabular}{|llllll|}
\hline Variable & $\begin{array}{l}\text { Area under the } \\
\text { ROC curve }\end{array}$ & $\begin{array}{l}\text { Standard } \\
\text { error }\end{array}$ & P-value & $95 \%$ confidence interval & \\
\cline { 1 - 2 } & +LR & & & & 0.989 \\
\hline Orosomucoid & 0.953 & 0.021 & 0.00 & 0.875 & 0.996 \\
\hline RBP & 0.970 & 0.022 & 0.00 & 0.900 & \\
\hline
\end{tabular}

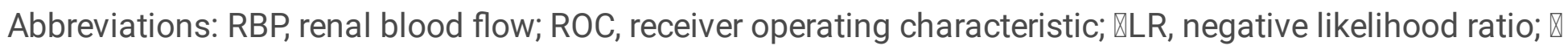
LR, positive likelihood ratio.

\section{Discussion}


DN has become the leading indication for dialysis due to end-stage renal disease (ESRD). (12) Recent findings suggest that immune-mediated inflammatory processes play a crucial role in DN. Many preinflammatory cells, growth regulators, and adhesion factors interact with each other and cross-link, resulting in expansion of the corresponding cascade of inflammation. (13) In recent years, the rapid development of proteomics technology has provided us with new methods and ideas for identifying early diagnostic markers of DN. Proteomics techniques have been used to identify disease-specific biomarkers and other related proteins in urine. Differential proteins have been identified, and some protein markers were found to have predictive effects on glomerular diseases.

Orosomucoid protein is a non-specific acute phase reaction protein that is mainly synthesized and secreted by the liver; it is low in healthy body fluids but is significantly increased in a state of inflammation or during tumor growth. Orosomucoid can act in damaged areas, be released into the circulation and intercellular fluid, and become involved in the induction and regulation of body damage, immune, and inflammatory responses. (14) Elevated urine orosomucoid levels in T2DM patients have predictive effects on cardiovascular complications and mortality. (15) El-Beblawy et al. (7) pointed out that orosomucoid is an independent factor for diabetic microvascular complications and can be considered an early marker of kidney damage. Fandiño-Vaquero et al. (16) also found that orosomucoid levels increased in patients with T2DM and might mirror local endothelial dysfunction or inflammatory processes. Although there are existing studies on orosomucoid, studies on changes in orosomucoid concentration in urine during the early stage of DN is lacking. In this study, we found that as the disease progressed, urine orosomucoid levels gradually increased $(P<0.001)$. Orosomucoid might be an independent risk factor for T2DM and T2DN, and it had a significant positive correlation with MAL ( $r=$ $0.489)$ and a significant negative correlation with eGFR $(r=-0.577)$. The results also revealed an increase in orosomucoid in the early stage of $\mathrm{DN}$, suggesting that this increase may promote the occurrence and development of DN.

RBP is filtered through the glomerulus and absorbed and degraded by proximal tubular epithelial cells. Therefore, it is generally stable in urine, difficult to decompose, and has a low excretion rate. An increase in RBP excretion may reflect the extent of damage. $(17,18)$ Studies have shown that urine RBP levels in patients with T2DM are closely related to DN. $(19,20)$ Some studies have shown that urine RBP can be used to assess the degree of renal interstitial fibrosis due to various causes, progress with ESRD dialysis, and even diabetes related to an increased risk of death. $(21,22)$ This study found that urine RBP and MAL levels in the T2DN group were significantly increased $(P<0.001)$. Owing to a significant positive correlation with MAL $(r=0.468)$ and a significant negative correlation with eGFR $(r=-0.474)$, RBP might be an independent risk factor for T2DN. Urine RBP may reflect early renal damage in DN. In the area under the ROC curve for predicting DN using orosomucoid and RBP, both factors had high sensitivity and specificity. Therefore, both orosomucoid and RBP can be used to diagnose DN.

This study's main limitation is that all the participants were residents of Henan Province, China, and the sample size was small. Hence, further verification is needed through large sample-size and multicenter studies. 


\section{Conclusions}

Urine orosomucoid and RBP can serve as markers for the early diagnosis of DN that would also aid in the timely treatment of DN. However, the underlying molecular mechanisms and the clinically important levels of these potential biomarkers need to be studied further.

\section{Declarations}

\section{Ethics approval and consent to participate}

The study protocol was approved by the ethics committee of The First Affiliated Hospital of Henan Polytechnic University (Jiaozuo Second People's Hospital) (IRB number: 2016010), and all patients provided written informed consent to participate in the study.

\section{Consent for publication}

Consent for publication was provided.

\section{Availability of data and materials}

The datasets used and analyzed during the current study are available from the corresponding author on reasonable request and with the permission of The First Affiliated Hospital of Henan Polytechnic University ethics committee.

\section{Competing interests}

The authors declare that they have no competing interests

\section{Funding}

This work was supported by the Jiaozuo City Medical Research Project under grant number $2017 \mathrm{KY} 018$. The funders had no role in the study design, data collection and analysis, decision to publish, or preparation of the manuscript.

\section{Authors' contributions}

SY $L$ analyzed and interpreted the patient data regarding diabetic nephropathy and the normal control. LF conducted serological testing and analysis. BY, YF L, XQ W, JC, and HH W screened the hospitalized patients and entered the patient data. XH Z was a major contributor in writing the manuscript. All authors have read and approved the final manuscript.

\section{Acknowledgments}

Not applicable. 


\section{Abbreviations}

2D-DIGE Two-dimensional fluorescence difference gel electrophoresis

2-DE Two-dimensional gel electrophoresis

AKI Acute kidney injury

BMI Body mass index

$\mathrm{CHOL} \quad$ Cholesterol

$\mathrm{Cl} \quad$ Confidence interval

DBP Diastolic blood pressure

DN Diabetic nephropathy

eGFR Estimated glomerular filtration rate

ESRD End-stage renal disease

FBG $\quad$ Fasting blood glucose

HbA1c glycosylated hemoglobin

LDL Low-density lipoprotein

MAL Microalbumin

NC Normal control

NGAL Neutrophil gelatinase-associated lipocalin

RBF Renal blood flow

RBP Retinol binding protein

ROC Receiver operating characteristic

SBP Systolic blood pressure

$\mathrm{SCr} \quad$ Serum creatinine

T2DM Type 2 diabetes mellitus

TG

Triglycerides 


\section{References}

1. Kapoula GV, Kontou PI, Bagos PG. Diagnostic accuracy of neutrophil gelatinase-associated lipocalin for predicting early diabetic nephropathy in patients with Type 1 and Type 2 Diabetes Mellitus: A systematic review and meta-analysis. J Appl Lab Med. 2019;4:78-94.

2. Furuichi K, Shimizu M, Hara A, Toyama T, Wada T. Diabetic nephropathy: A comparison of the clinical and pathological features between the CKD risk classification and the classification of diabetic nephropathy 2014 in Japan. Intern Med. 2018;57:3345-50.

3. Papadopoulou-Marketou N, Kanaka-Gantenbein C, Marketos N, Chrousos GP, Papassotiriou I. Biomarkers of diabetic nephropathy: A 2017 update. Crit Rev Clin Lab Sci. 2017;54:326-42.

4. Varghese SA, Powell TB, Budisavljevic MN, Oates JC, Raymond JR, Almeida JS, et al. Urine biomarkers predict the cause of glomerular disease. Am Soc Nephrol. 2007;18:913-22.

5. Lewandowicz A, Bakun M, Kohutnicki R, Fabijanska A, Kistowski M, Imiela J, et al. Changes in urine proteome accompanying diabetic nephropathy progression. Pol Arch Med Wewn. 2015;125:27-38.

6. Jang H. Type 2 diabetic nephropathy urine proteomics research and early diagnosis marker screening. 2009 Shandong University, PhD dissertation.

7. El-Beblawy NM, Andrawes NG, Ismail EA, Enany BE, El-Seoud HS, Erfan MA. Serum and urinary orosomucoid in young patients with type 1 diabetes: a link between inflammation, microvascular complications, and subclinical atherosclerosis. Clin Appl Thromb Hemost. 2016;22:718-26.

8. Wang $H$, Bao X, Ma Y, Shan X, Huang C. Urinary orosomucoid 1 protein to creatinine ratio as a potential biomarker for early screening of kidney impairment in type-2 diabetes patients. Nephrology. 2020;25:667-75.

9. Mahfouz MH, Assiri AM, Mukhtar MH. Assessment of neutrophil gelatinase-associated lipocalin (NGAL) and retinol-binding protein 4 (RBP4) in type 2 diabetic patients with nephropathy. Biomark Insights. 2016;11:31-40.

10. Roden M. [Diabetes mellitus: definition, classification and diagnosis]. Wien Klin Wochenschr. 2016;128:S37-S40. [Article in German]

11. Schwandt A, Denkinger M, Fasching P, Pfeifer M, Wagner C, Weiland J, et al. Comparison of MDRD, CKD-EPI, and Cockcroft-Gault equation in relation to measured glomerular filtration rate among a large cohort with diabetes. J Diabetes Complications. 2017;31:1376-83.

12. Nichols GA, Vupputuri S, Lau H. Medical care costs associated with progression of diabetic nephropathy. Diabetes Care. 2011;34:2374-78.

13. Liu SY, Chen J, Li YF. Clinical significance of serum interleukin-8 and soluble tumor necrosis factorlike weak inducer of apoptosis levels in patients with diabetic nephropathy. J Diabetes Investig. 2018;9:1182-8.

14. Luo Z, Lei H, Sun Y, Liu X, Su DF. Orosomucoid, an acute response protein with multiple modulating activities. J Physiol Biochem. 2015;7:329-40. 
15. Lage R, Moscoso I, Fernández-Trasancos Á, Cebro M, Couselo M, Fandiño-Vaquero R, et al. Differential behaviour of epicardial adipose tissue-secretomes with high and low orosomucoid levels from patients with cardiovascular disease in H9C2 cells. Mol Cell Endocrinol. 2015;416:77-87.

16. Fandiño-Vaquero R, Fernández-Trasancos A, Alvarez E, Ahmad S, Batista-Oliveira AL, Adrio B, et al. Orosomucoid secretion levels by epicardial adipose tissue as possible indicator of endothelial dysfunction in diabetes mellitus or inflammation in coronary artery disease. Atherosclerosis. 2014;235:281-8.

17. Andreucci M, Faga T, Pisani A, Perticone M, Michael A. The ischemic/nephrotoxic acute kidney injury and the use of renal biomarkers in clinical practice. Eur J Intern Med. 2017;39:1-8.

18. Adiyanti SS, Loho T. Acute Kidney Injury (AKI) biomarker. Acta Med Indones 2012;44:246-55.

19. Klisic A, Kavaric N, Ninic A. Retinol-binding protein 4 versus albuminuria as predictors of estimated glomerular filtration rate decline in patients with type 2 diabetes. J Res Med Sci. 2018;23:44.

20. Ni X, Gu Y, Yu H, Wang S, Chen Y, Wang X, et al. Serum adipocyte fatty acid-binding protein 4 levels are independently associated with radioisotope glomerular filtration rate in type 2 diabetic patients with early diabetic nephropathy. Biomed Res Int. 2018;2018:4578140.

21. Pallet $N$, Chauvet $S$, Chassé JF, Vincent M, Avillach P, Levi C, et al. Urinary retinol binding protein is a marker of the extent of interstitial kidney fibrosis. PLoS One. 2014;9:e84708.

22. Wu J, Shao X, Lu K, Zhou J, Ren M, Xie X, et al. Urinary RBP and NGAL levels are associated with nephropathy in patients with type 2 diabetes. Cell Physiol Biochem. 2017;42:594-602.

\section{Figures}




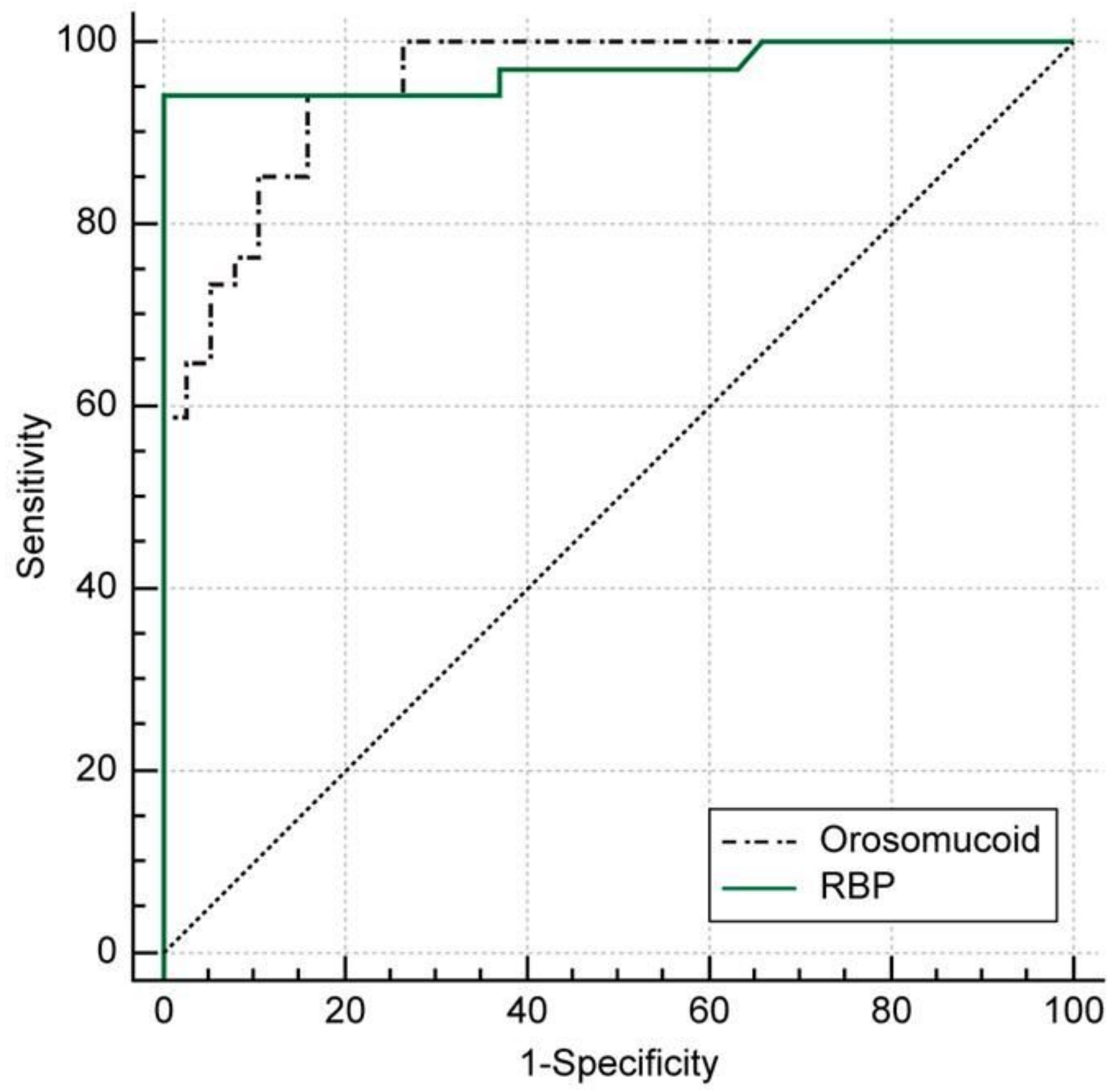

Figure 1

Receiver operating characteristic curve of two indicators for predicting diabetic nephropathy RBP: retinol binding protein 